International Journal of Engineering \&Technology, $7(3.12)(2018) 734-736$
International Journal of Engineering \& Technology
SPC
Website: www.sciencepubco.com/index.php/IJET
Research paper

\title{
Performance Enhancement and IoT Based Controlling for Smart Home
}

\author{
S.Kayalvizhi $^{1^{*}}$, K. Pradeep Kumar ${ }^{2}$, S. Sai Akhil ${ }^{3}$, T. Vinay ${ }^{4}$ \\ Assistant Professor ${ }^{1}, U G$ student ${ }^{2,3,4}$ \\ Department of ECE, SRM Institute of Science and Technology \\ *Corresponding Author E-mail: kayalvizhi.s@ktr.srmuniv.ac.in ${ }^{I}$
}

\begin{abstract}
Automation is becoming vastly used due to its numerous advantages. Building automation refers to control any type of building appliances by using mobile network or the local area network. It is a process of operating or controlling electronic appliances by reducing the basic human requirments.
\end{abstract}

\section{Introduction}

The popularity of network embedded home systems is increasing the past few years as of the simplicity and affordability rates. More power-full and faster processors are being manufactured which can be Incorporated into our day to day tools and devices. With the fast develop-ment of the Internet administrations, there is the possibility for the checking and remote monitoring of such system empowered machines. Moreover, the new and energizing chances to increment the network of gadgets inside the home condition with the end goal of home mechanization through web are yet to be investigated. In the recent years noteworthy research into the field of home computerization along with usage of many other possible communication methods that are more efficient and reliable. In addition to this the home centralization systems are being designed not only to maintain the household devices but also to monitor the health resources. These home automation devices are combined with $\mathrm{AI}$ and cloud computing to make them not only faster but also more responsive to environmental or human conditional changes. These devices are Incorporated with different types of devices along with body sensors. Features of proposed system

This paper presents a novel approach on the home automa-tion system making it simplified and reliable. In this paper we are going to incorporate all the devices used in home to the arduino rather than raspberry pi the the previous models and connecting every individual model through arduino. By using and incorporating it to a single device we can make a centralized system which can easily control all the other devices. The previous model has a design flaw that can be used to hack into the system and let anyone control the devices but in this model we are using an authentication page to access the home automation system and to control it. By this way we can update the system through the internet if there are any bugs in the program and also keep it secure for usage. The power used for by the whole system will be very low so it can keep on running without any heating issues.

\section{System Architecture}

This System is build based on Arduino and the remain-ing modules are connected directly connected to Arduino and are controlled by it. We can connect any IoT device that can be electrically controlled and monitored. We are using a Wi-Fi module to connect the arduino to the internet. A web server is also built so that user can connect and control the devices through a GUI application.

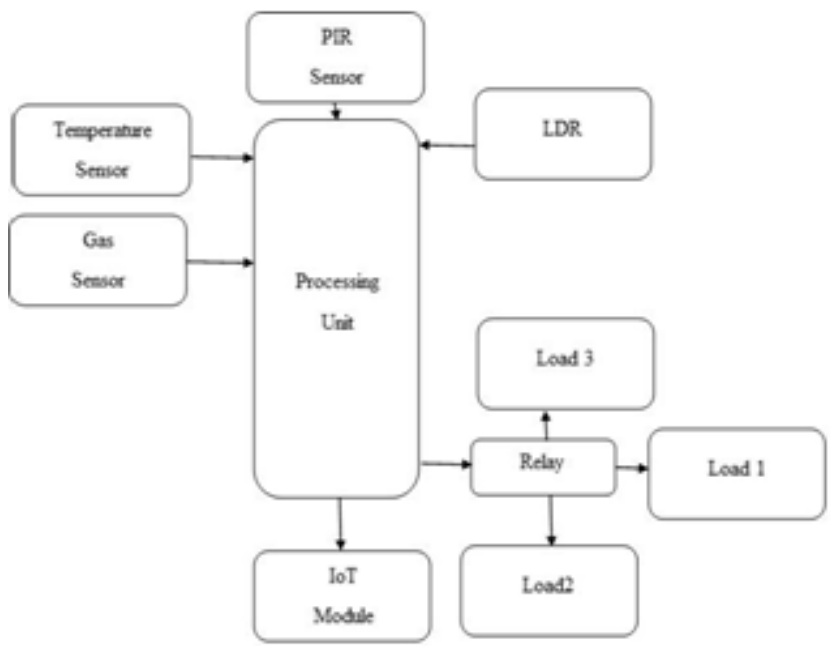

Fig. 1: Block

\section{Hardware}

\section{Arduino}


It is an open-source computer hardware and soft-ware as-sociation, backed up by the user community that builds and manufactures single-board micro-controllers and micro-controller kits for building digital devices and interactive objects that can sense and control objects in the physical world.

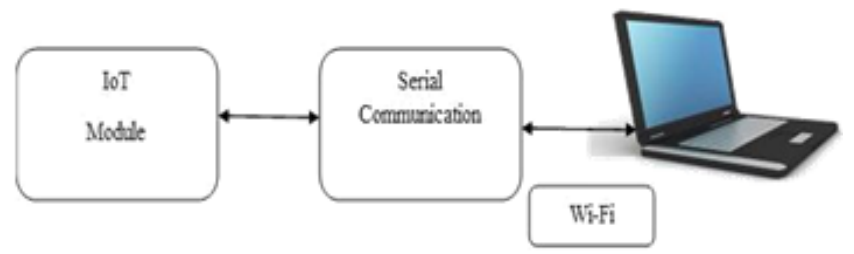

Fig. 2: Communication

the SMS (Short Message Service). It can be used with a $12 \mathrm{v}$ battery or a $9 \mathrm{v}$ battery. In this article we are going to test one of the cheapest and easy to use GSM development platform, the SIM 900A arduino compatible module. The SIM 900A is a low cost and with full TCP/IP capability, integrated with the I/O digital pins which can be controlled via pseudo-code like programming code language.

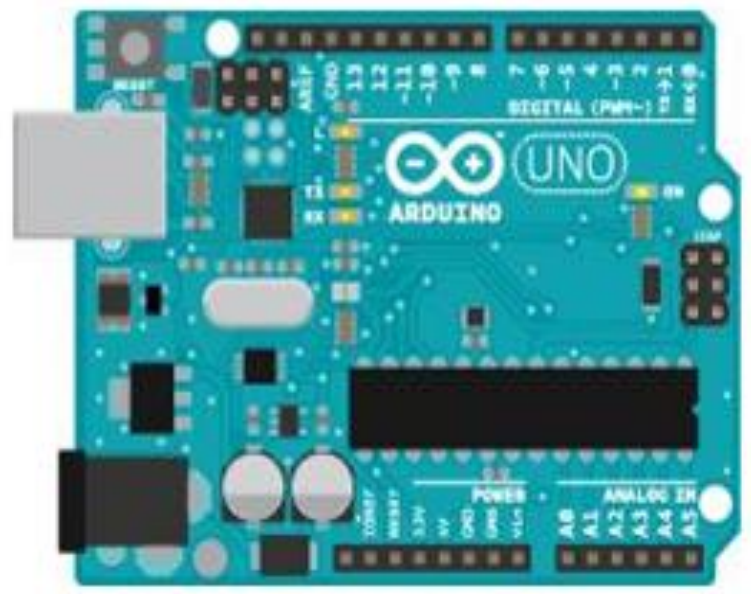

Fig. 3: Arduino

\section{LDR}

It is a component that can vary it's resistance when diferrent intensity of light falls upon it. This is very useful to use in the light sensing and light sensitive circuits for various purposes.

\section{LED}

A light-emitting diode (LED) is a two lead semiconductor light source. It is embedded with a p-n junction diode which emits light when when electricity is given to both ends of the diode. When a threshold current is given to the leads, electrons are able to recombine with electron-holes inside the device, releasing energy in the form of photons which is light. This effect is called electro luminescence, and the colour of the light (correspond-ing to the energy of the photon) is determined by the energy band gap of the semiconductor. LED are very small (less than $1 \mathrm{~mm} 2$ ) and incorperated optical components may be used to shape the radiation pattern.

\section{Gas Sensor}

A gas sensor is a device that detects the manifestation of different gases in an area, used mostly as part of a safety system. This type of instrument can be used to detect a gas leak or other emissions and can used by the sensor values to detect the gas leak and process the information and process the next step in the program. A gas detector can send notification to operators in the area where there is a leak allowing to take the necessary action. This type of device is very important as there are many reported incidents that occured due to the gas leak that are harmfull to biological life.

\section{GSM Module}

GSM (Global System for Mobile Communications) module which can access the mobile networks with the insertion of the SIM (Subscriber Identity Module) card which contains the information of the user to connect to the operator that is being used. It is used to access the internet or to send

\section{Interface and Results}

The Arduino is controlled through the internet using a web based GUI. For security, a user name and password is used. All the devices are controlled form using this web page. The arduino also reads the values that are being generated by the devices and displays them at set time intervals.

\begin{tabular}{|l|l|l|l|}
\hline Date & Temp & Gas & Lodr \\
\hline April 6, 2018, 10:18 am & 50 & 87 & 973 \\
\hline April 6, 2018, 10:19 am & 47 & 82 & 979 \\
\hline April 6, 2018, 10:20 am & 56 & 91 & 979 \\
\hline
\end{tabular}

Fig. 4: Values

The devices are controlled by using a GUI buttons on the same web page. These might be any of the devices connected and configured with the arduino. There is also a variable graph that is show in the web page so that we can see the overall changes that are formed in the environment.

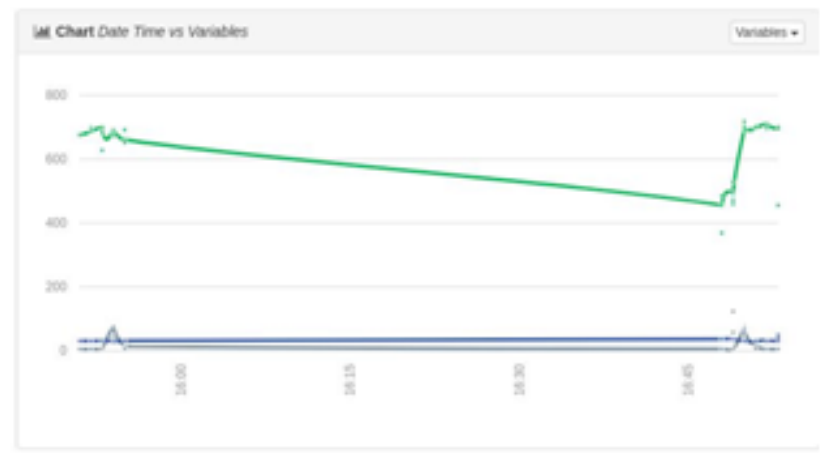

Fig. 5: Variable

We can also receive notification at set intervals or if there is any emergency at the house. We will also get a notification if the sensor values cross the threshold values. These alerts are necessary when there is any fire incident or if there is any other emergency like breakin or other. 


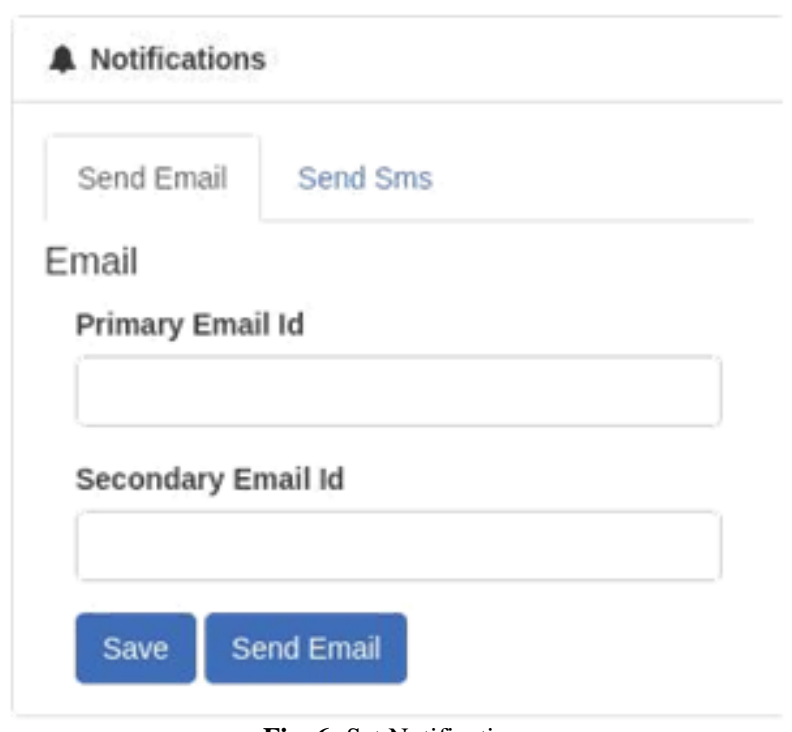

Fig. 6: Set Notifications

We can either receive the notification on the given email or the given mobile number these are usefull when we dont have either of the devices with us and are using the other ones.

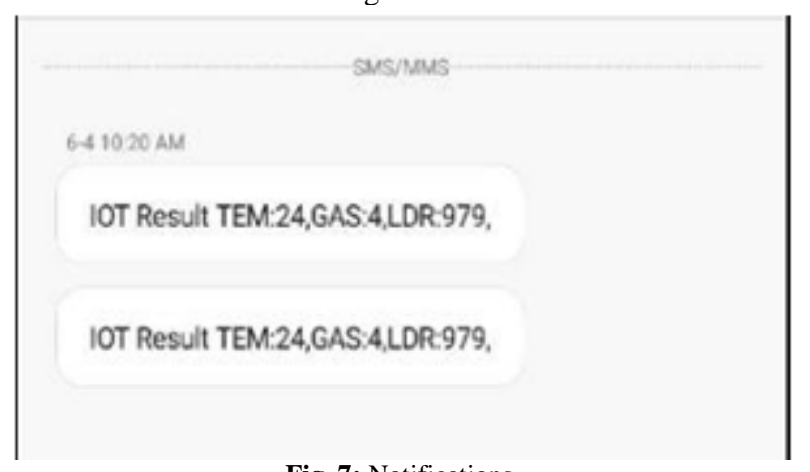

Fig. 7: Notifications

\section{Conclusion}

This study proposes a smart home-automation which uses smart interface to interact with the user. In addition to integrating smart AI along with cloud computing to offload the processing required to the online web severs we can speed up the process and the time required to complete the code cycles. Many more advanced and smaller devices can be developed and employed onto the user itself. The power consumption of the core devices used will be far low that it can basically run on a $12 \mathrm{v}$ battery making it completely independant to the power supply.

\section{References}

[1] H. Ardam and I. Coskun, "A remote controller for home and office appliances by telephone", IEEE Transactions on Consumer Electronics, vol. 44, no. 4, pp. 1291-1297, 1998.

[2] K. Bromley, M. Perry, and G. Webb. "Trends in Smart Home Systems, Connectivity and Services", www.nextwave.org.uk, 2003.

[3] A. R. Al-Ali and M. Al-Rousan, "Java-based home automation system", IEEE Transactions on Consumer Electronics, vol. 50, no. 2, pp. 498504, 2004.

[4] S. Ok and H. Park, "Implementation of initial provisioning function for home gateway based on open service gateway initiative platform", The 8th International Conference on Advanced Communication Technology, pp. 1517- 1520, 2006.
[5] D. Yoon, D. Bae, H. Ko and H. Kim, "Implementation of Home Gateway and GUI for Control the Home Appliance", The 9th International Conference on Advanced Communication Technology, pp. 15831586, 2007.

[6] Khusvinder Gill, Shuang-Hua Yang, Fang Yao, and Xin Lu, ”A ZigBee- Based Home Automation System", IEEE Transactions on Consumer Electronics, Vol. 55, No. 2, MAY 2009. ing-Tsung Lee, Wei-Hsuan Hsiao, Chin-Meng Huang and Seng-Cho T. Chou, "An Integrated Cloud-Based Smart Home Management System with Community Hierarchy", IEEE Transactions on Consumer Electronics, Vol. 62, No. 1, February 2016. 